\title{
Marfanoid hypermobility syndrome associated with coarctation of the aorta
}

\author{
A. DANESHWAR, D. TAVAKOLI, AND J. NAZARIAN \\ From National Heart Centre, University of Azarabadegan, Tabriz, Iran
}

SUMMARY There are intimate interrelations between various elements of connective tissue, viz. collagen, elastin, and glycoproteins. It is not unexpected that Marfan and Ehlers-Danlos syndromes share common features. The condition is labelled as Marfanoid hypermobility syndrome. In the patient described here, the Marfanoid hypermobility syndrome was associated with coarctation of the aorta which was corrected surgically.

Ehlers-Danlos syndrome is characterised by hypermobility of joints, hyperextensibility of skin, defective healing, and easy bruising (McKusick, 1972a), while the usual features of the Marfan syndrome are long limbs, arachnodactyly, a high arched palate, and skeletal deformity, particularly spinal scoliosis and pectus excavatum. Ectopia lentis is present in 70 per cent of patients with Marfan's syndrome but the skin is normal except for the common presence of striae (McKusick, 1972b).

Because there are intimate interrelations between various elements of connective tissue, i.e. collagen, elastin, and glycoproteins, it is not unexpected that Marfan and Ehlers-Danlos syndromes, two inherited connective disorders, may share common features. Walker et al. (1969) described a patient whose body habitus was typical of the Marfan syndrome but who also had the gross joint hypermobility and cutaneous hyperextensibility of the Ehlers-Danlos syndrome and these authors labelled the condition as 'Marfanoid hypermobility syndrome'. Cotton and Brandt (1976) have described two cases of Marfanoid hypermobility syndrome, one associated with 'floppy' mitral valve and the other with cystic medionecrosis of the aorta. The unusual association of coarctation of the aorta and Marfanoid hypermobility syndrome, which was seen in the present case and has not been documented previously, has prompted the publication of this report.

\section{Case report}

A 15-year-old boy was admitted to the Pahlavi Hospital, Tabriz, Iran, with complaints of diplopia and blurring of vision of 4 months' duration. He also had praecordial pain, radiating to the left shoulder and back, pain in the calf muscles on both sides, and severe headache during exercise of the same duration. He gave a history of occasional episodes of epistaxis and difficult healing of wounds following injuries. There was no family history of dolichomorphism, eye disorders, bleeding, abnormal skin, defective wound healing, hypermobility of joints, or sudden death.

Examination disclosed a young boy of thin build with height of $172 \mathrm{~cm}$ and arm span of $178 \mathrm{~cm}$. His upper : lower segment ratio was 0.92 (normal range $0.93 \pm 0.08$ ). His weight was $50.4 \mathrm{~kg}$ and blood pressure was $145 / 100 \mathrm{mmHg}$ in both upper extremities in the erect and supine positions. Blood pressure in the lower extremities could not be recorded as the arterial pulsations in them were too feeble. There were pulsations of the carotid arteries in the neck and of collaterals over the back, adjacent to the scapulae. Murmurs were heard over these pulsating vessels. He had a high arched palate. His hands were narrow with thin elongated fingers.

His skin was hyperplastic (Fig. 1A) and epicanthal folds were present. The joints of the hands and feet 

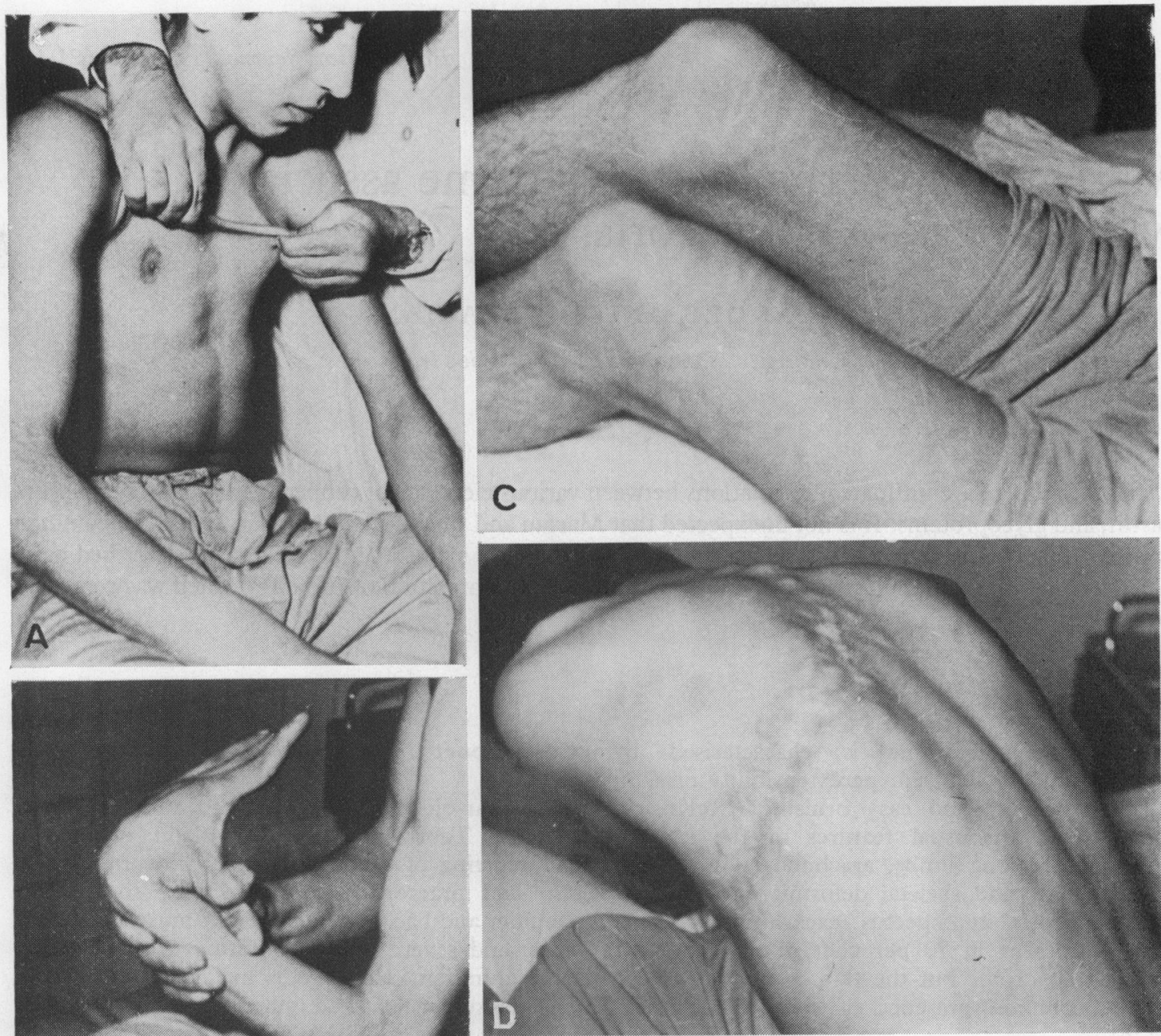

Fig. 1 (A) Hyperelasticity of skin; (B) hypermobility of joints of hand; $(C)$ multiple white striae over knees; $(D)$ wide paper-like operation scar.

could be hyperextended (Fig. 1B). The skin over the knee joints showed white striae (Fig. 1C). There were many cigarette paper scars of healed wounds over the body. There was no abnormality of the respiratory system. Ophthalmic examination was normal.

The patient's haemoglobin was $14 \mathrm{~g} / \mathrm{dl}$ and coagulation studies of the blood were normal. The urinalysis and cytogenetic studies did not show any abnormality. The $x$-ray film of the chest showed notching of ribs, left ventricular hypertrophy, and a positive ' 3 ' sign.

He was operated on for coarctation of the aorta. There was distinct bleeding during the operation and haemostasis was obtained with difficulty. The stenosis was postductal. The stenotic portion 
was resected and replaced with 'dacron' graft. The postoperative course was uneventful. The wound healed with a wide paper-like scar (Fig. 1D). One month after operation his blood pressure was $120 / 85 \mathrm{mmHg}$ in the upper extremities and $100 / 60 \mathrm{mmHg}$ in the lower extremities, with good peripheral pulsations.

\section{Discussion}

Patients with features of both Marfan and EhlersDanlos syndromes have been reported in the literature (Goodman et al., 1965; Walker et al., 1969; Birkenstock et al., 1973; Motoyoshi et al., 1973; Cotton and Brandt, 1976) and these authors labelled this condition as a separate entity, the Marfanoid hypermobility syndrome. The hallmark of Marfan syndrome is dolichostenomelia. The cutaneous hyperextensibility and paper scars, the chief features of Ehlers-Danlos syndrome, are not usually present in Marfan's syndrome. The present case had arachnodactyly, high arch palate, and white skin striae, which are characteristic of the Marfan syndrome, and he also had hyperextensibility of the skin, paper scars, and hypermobility of the joints of the hand and feet, which are features of Ehlers-Danlos syndrome and, thus, he can be considered to be another example of the Marfanoid hypermobility syndrome. Cotton and Brandt (1976) have described cardiovascular abnormalities in 2 cases of Marfanoid hypermobility syndrome, one had 'floppy' mitral valve and the other presented with aortic aneurysm caused by cystic medionecrosis of the aorta. The present case had associated coarctation of the aorta which has not been described previously.

Ehlers-Danlos syndrome has been subdivided into 7 subgroups on the basis of clinical and genetic differences. A specific biochemical defect has been identified in 3 of these subgroups. The nature of the basic defect in Marfan's syndrome is not clear.
Goodman et al. (1965) and Motoyoshi et al. (1973) found autosomal dominant transmission of both the Ehlers-Danlos and Marfan syndromes in several family members of a patient, with features of both Ehlers-Danlos and Marfan syndromes and have raised the possibility that the two conditions are inherited simultaneously. The lack of evidence of either the Ehlers-Danlos or Marfan syndrome in the relatives of the present patient and those reported by Walker et al. (1969) and Cotton and Brandt (1976) makes it unlikely that these patients inherited two generalised connective tissue disorders, and they appear to have resulted from a spontaneous mutation.

\section{References}

Birkenstock, W. E., Louw, J. W., Maze, A., and Sladen, R. N. (1973). Combined Ehlers-Danlos and Marfan's syndromes. South African Medical fournal, 47, 2097-2102.

Cotton, D. J., and Brandt, K. D. (1976). Cardiovascular abnormalities in the Marfanoid hypermobility syndrome. Arthritis and Rheumatism, 19, 763-768.

Goodman, R. M., Wooley, C. F., Frazier, R. L., and Covault, L. (1965). Ehlers-Danlos syndrome occurring together with the Marfan syndrome; report of a case with other family members affected. New England fournal of Medicine, 273, 514-519.

McKusick, V. A. (1972a). The Ehlers-Danlos syndrome. In Heritable Disorders of Connective Tissue, 4th edn., pp. 292-371. C. V. Mosby, Saint Louis.

McKusick, V. A. (1972b). The Marfan syndrome. In Heritable Disorders of Connective Tissue, 4th edn., pp. 61-223. C. V. Mosby, Saint Louis.

Motoyoshi, K., Momoi, H., Mikami, R., et al. (1973). A family with many members of the marfanoid hypermobility syndrome. Fournal of fapan Society of Internal Medicine, 62, 23-29.

Walker, B. A., Beighton, P. H., and Murdock, J. L. (1969). The Marfanoid hypermobility syndrome. Annals of Internal Medicine, 71, 349-352.

Requests for reprints to Professor A. Daneshwar, National Heart Centre, Faculty of Medicine, University of Azarabadegan, Tabriz, Iran. 